\title{
Disfunciones sexuales femeninas en la comunidad de Sayausí, Cuenca 2019
}

\author{
Female sexual dysfunctions in the community of Sayausí, \\ Cuenca 2019
}

\section{Pinos Vélez Erika Aracely', Picón Rodríguez Jennifer Pamela', Tapia Cárdenas Jeanneth Patricia²}

VOLUMEN 39 | No 1 | SEPTIEMBRE 2021

FECHA DE RECEPCIÓN: 12/03/2021 FECHA DE APROBACIÓN: 06/10/2021 FECHA PUBLICACIÓN: 06/10/2021

1. Médica en libre ejercicio. Cuenca - Ecuador

2. Universidad de Cuenca. Centro de Postgrado. Especialización en Ginecología y Obstetricia. Cuenca-Ecuador

\section{Artículo Original original Article}

DOI: https://doi.org/10.18537/RFCM.39.01.04

Correspondencia: eapv0612ahotmail.com

Dirección:

Yanaurco y Río Upano

Código Postal:

010113

Celular:

0987779206

Cuenca - Ecuador

\section{RESUMEN}

Objetivo: determinar la prevalencia y factores asociados a la disfunción sexual femenina en la comunidad de Sayausí, periodo mayo-septiembre del 2019.

Metodología: estudio descriptivo y de corte transversal. Se aplicó una encuesta a 253 pacientes obtenidos por muestro probabilístico que cumplieron los criterios de inclusión. El análisis se realizó mediante estadística descriptiva, utilizando el programa SPSS versión 20. La investigación fue aprobada por el Comité Institucional de Bioética de la Universidad Católica.

Resultados: la prevalencia de disfunción sexual femenina fue de $58.1 \%$. En cuanto a la función sexual por dominios, la alteración del deseo se presentó en un $70 \%$ de los casos, seguido del dominio de excitación con $41.9 \%$ y el orgasmo con $37.2 \%$.

Conclusiones: más de la mitad de las encuestadas tuvieron disfunción sexual según los puntajes del Índice de Función Sexual Femenina.

Palabras clave: sexualidad, relaciones sexuales, orgasmo, dispareunia.

\section{ABSTRACT}

Objective: to determine the prevalence and factors associated with female sexual dysfunction in the community of Sayausí, during the period MaySeptember 2019.

Methodology: it is a descriptive and cross-sectional study. A survey by probabilistic sampling was applied to 253 patients who met the inclusion criteria. The analysis was carried out through descriptive statistics, using the SPSS version 20 program. The research was approved by the Institutional Committee of Bioethics of the Catholic University.

Results: the prevalence of female sexual dysfunction was $58.1 \%$. Regarding sexual function by domains, the alteration of desire was presented in $70 \%$ of the cases, followed by the excitement domain with $41.9 \%$ and orgasm with $37.2 \%$.

Conclusions: more than half of the respondents had sexual dysfunction according to the scores of the Female Sexual Function Index.

Key words: sexuality, unsafe sex, orgasm, dyspareunia. 


\section{INTRODUCCIÓN}

La sexualidad femenina es sin duda un referente de la calidad de vida, pues en un sentido estrictamente pragmático es parte fundamental de su salud, cuyo correcto funcionamiento va en armonía con el completo bienestar físico, psicológico y social; además, la sexualidad femenina posee una mayor plasticidad erótica que la masculina, ya que debe soportar una fuerte presión en estándares de belleza y atractivo sexual ${ }^{1,2}$.

Las disfunciones sexuales femeninas (DSF), son un problema latente a través de los tiempos, cuya génesis y consecuencias han tenido poca o nula atención ${ }^{3,4}$, no obstante, hoy en día estas patologías son consideradas como trastornos de orden heterogéneo ${ }^{5}$ que repercuten en la capacidad que tiene una persona en su repuesta y satisfacción sexual, visualizándose alteraciones en cualquiera de sus etapas tales como: el deseo, la excitación y el orgasmo ${ }^{6}$.

La visión evidentemente positivista de la medicina en la práctica sanitaria, ha dificultado el abordaje adecuado de las $\mathrm{DSF}^{7,8}$, siendo apreciable la falta de iniciativa tanto del médico como del paciente, en abordar temas que impliquen campos sexuales, creando así un desconocimiento sobre el tema lo que conlleva a una dificultad en su prevención, diagnóstico y/o manejo terapéutico ${ }^{1,2}$.

Se define como disfunción sexual a la incapacidad o dificultad de participar en las relaciones sexuales tal como lo desea el individuo9. La DSF puede implicar efectos negativos sobre la salud y la calidad de vida de una mujer; reflejando una interrelación de eventos interpersonales, psicosociales y anímicos, los cuales a su vez son influenciados por factores psicológicos, genéticos, hormonales, anatómicos y endocrinos ${ }^{10,11}$.

El estudio de la respuesta sexual se inicia a partir del año 1950 con el autor Kinsey; posteriormente en la década de 1960, Masters y Johnson, estudiaron un ciclo de respuesta sexual en cuatro fases o etapas: excitación, meseta, orgasmo y resolución. En 1970, Kaplan lo modificó a un modelo con solo tres fases: deseo, excitación y orgasmo; no obstante, la importancia de estos dos modelos era que el orgasmo se considera esencial para el bienestar sexual. Por otro lado Basson, propuso un modelo del cual la satisfacción sexual es el principal objetivo de la relación sexual ${ }^{12,13}$. Las fases que son fundamentales en la fisiología del acto sexual son:

Excitación: esta fase se caracteriza por la actividad que ejerce el sistema nervioso parasimpático, en la cual se aumenta la tensión muscular y la frecuencia cardíaca, a nivel de los órganos sexuales se producen: vasodilatación de arteriolas perivaginales, agrandamiento del clítoris, expansión del barril vaginal; y, por último, gracias al efecto de los estrógenos se concreta la lubricación vaginal, esta fase puede durar desde algunos minutos hasta algunas horas ${ }^{14,15}$.

Meseta: se caracteriza por ser el máximo nivel de excitación alcanzado y que se mantiene antes de llegar al orgasmo, a nivel de los órganos genitales se produce una expansión y congestión con sangre a nivel vaginal, que se define como una plataforma orgásmica ${ }^{13}$.

Orgasmo: durante la fase orgásmica se producen contracciones musculares involuntarias, de varios grupos musculares genitales; además, existe aumento de la frecuencia respiratoria, cardíaca y de la presión arterial, para culminar con una liberación de la tensión nerviosa y una experiencia placentera $^{13-16}$.

Resolución: después de la fase orgásmica continúa la etapa de resolución, donde el cuerpo vuelve a un estado no excitado, con valores normales de la frecuencia cardíaca, respiración y presión arterial; muchas veces puede observarse el periodo refractario y puede experimentar múltiples orgasmos poco después del primero ${ }^{13-16}$.

La clasificación que se utiliza actualmente, abarca tres categorías que son: el trastorno del deseo sexual hipoactivo, de excitación femenina el cual se ha asociado a la categoría de trastorno por interés o excitación de la mujer, al igual que la dispareunia y el vaginismo en la categoría de dolor genitopélvico; por último, el trastorno orgásmico, el cual no ha presentado ningún cambio $^{17,18}$.

\section{METODOLOGÍA}

Estudio de tipo descriptivo, de cohorte transversal. La investigación se realizó en el Centro de Salud 
Sayausí, perteneciente al Distrito 01D02 de la Zona de Salud 6 del Ecuador. La población estuvo constituida por mujeres que acudieron para atención en consulta externa de dicha institución durante el periodo mayo-septiembre del 2019.

El muestreo fue no probabilístico por conveniencia, participaron aquellas pacientes que acudieron para atención médica en la institución durante el periodo especificado, se incluyeron a mujeres con edades $\geq 18$ años que reportaron actividad sexual durante las últimas 4 semanas, previas a la aplicación de la encuesta y que firmaron el consentimiento informado. El instrumento de recolección de datos incluyó variables sociodemográficas. Para la recolección de la información de nivel socioeconómico e Índice de Función Sexual Femenina (IFSF) se aplicaron formularios validados. Los datos fueron procesados en el programa SPSS versión 20 .

La Escala de Graffar-Mendez Castellanos, instrumento que categorizó a las pacientes en 5 niveles, referenciados según la puntuación obtenida de 4 parámetros como: la ocupación del jefe de la familia, la instrucción de la madre, la fuente de ingresos económicos y las condiciones de la vivienda; en base a lo cual, se determinó una escala de puntuación: de 4 a 6 alto nivel económico, 7 a 9 medio-alto, 10 a 12 mediomedio, 13 a 16 pobreza relativa y de 17 a 20 pobreza extrema ${ }^{19}$.

El Índice de Función Sexual Femenina (IFSF), es una escala multidimensional, con propiedades psicométricas, ampliamente aplicada y validada en español y útil para evaluar la función sexual femenina, contó con 19 preguntas que evaluaron 6 dominios a saber: deseo, excitación, lubricación, orgasmo, satisfacción y dolor; donde cada uno de los items de preguntas contenía 5 a 6 opciones de respuesta, con una escala de valoración de 0 a 5 puntos; una vez que se concluyó con el llenado del formulario el puntaje que correspondió a cada dominio se multiplicó por una constante, luego se realizó la sumatoria de todos los dominios, interpretándose en la puntuación general de manera que si el puntaje era inferior o igual a 26.55 o cuando si la puntuación de cada dominio fue menor a 3.66 , se consideró criterio para disfunción sexual ${ }^{20}$.

\section{RESULTADOS}

Tabla $N^{\circ} 1$

Caracterización sociodemográfica de 253 pacientes atendidas en el Centro de Salud Sayausí, año 2019

\begin{tabular}{|c|c|c|}
\hline Variables & $\mathbf{n}$ & $\%$ \\
\hline \multicolumn{3}{|l|}{ Edad $^{*}$} \\
\hline$\leq 19$ años & 18 & 7.1 \\
\hline 20 a 39 años & 165 & 65.2 \\
\hline 40 a 64 años & 69 & 27.3 \\
\hline$\geq 65$ años & 1 & 0.4 \\
\hline \multicolumn{3}{|l|}{ Residencia } \\
\hline Urbana & 135 & 53.4 \\
\hline Rural & 118 & 46.6 \\
\hline \multicolumn{3}{|l|}{ Estado civil } \\
\hline Soltera & 84 & 33.2 \\
\hline Casada & 75 & 29.6 \\
\hline Viuda & 74 & 29.2 \\
\hline Divorciada & 20 & 7.9 \\
\hline \multicolumn{3}{|l|}{ Religión } \\
\hline Católica & 215 & 85 \\
\hline Evangélica & 12 & 4.7 \\
\hline Testigo de Jehová & 3 & 1.2 \\
\hline Ninguna & 19 & 7.5 \\
\hline Otras & 4 & 1.6 \\
\hline \multicolumn{3}{|l|}{ Ocupación } \\
\hline Trabaja & 102 & 40.3 \\
\hline Estudia & 90 & 35.6 \\
\hline Desempleados & 61 & 24.1 \\
\hline \multicolumn{3}{|l|}{ Instrucción } \\
\hline Ninguno & 4 & 1.6 \\
\hline Primaria & 52 & 20.6 \\
\hline Secundaria & 123 & 48.6 \\
\hline Superior & 74 & 29.2 \\
\hline \multicolumn{3}{|l|}{ Nivel socioeconómico } \\
\hline Alto & 14 & 5.5 \\
\hline Medio-alto & 62 & 24.5 \\
\hline Medio-medio & 87 & 34.4 \\
\hline Pobreza relativa & 87 & 34.4 \\
\hline Pobreza extrema & 3 & 1.2 \\
\hline
\end{tabular}


Se encuestaron 253 pacientes, de las cuales, según características sociodemográficas, más de la mitad de ellas tuvieron rangos de edad de entre 20 a 39 años, eran residentes en áreas urbanas, de estado civil solteras, religión católica.
Alrededor de la mitad de encuestadas trabajaba y refirió tener instrucción secundaria y en cuanto al nivel socioeconómico en su mayoría fueron clasificadas en un nivel socioeconómico medio y bajo (Tabla $N^{\circ} 1$ ).

\section{Tabla $\mathrm{N}^{\circ} 2$}

Distribución según características obstétricas e historia sexual de 253 pacientes atendidas en el Centro de Salud Sayausí, año 2019

\begin{tabular}{lcc}
\hline Variables & $\overline{\mathrm{X}}^{*}$ & DS $^{\star *}$ \\
\hline Edad inicio de vida sexual & 19 & 3 \\
Gestas & 2 & 1 \\
Partos & 2 & 1 \\
Compañeros sexuales & 2 & 1 \\
\hline
\end{tabular}

${ }^{*} \overline{\mathrm{x}}$ : promedio ${ }^{* *} \mathrm{DS}$ : desviación estándar

La media de edad del inicio de vida sexual fue de 19 años con una desviación estándar (DS) de \pm 3 , con una media de compañeros sexuales de 2 y una DS de \pm 1 ; por su parte la media de gestas y partos fue de 2 con una desviación estándar de \pm 1 (Tabla Nㅜ2).

\section{Tabla $\mathrm{N}^{\circ} 3$}

Puntaje de índice de función sexual femenina de 253 pacientes atendidas en el Centro de Salud de Sayausí, año 2019

\begin{tabular}{|c|c|c|c|c|c|c|c|c|c|c|c|c|c|c|}
\hline & \multicolumn{2}{|c|}{ Deseo } & \multicolumn{2}{|c|}{$\begin{array}{l}\text { Excita- } \\
\text { ción }\end{array}$} & \multicolumn{2}{|c|}{$\begin{array}{l}\text { Lubrica- } \\
\text { ción }\end{array}$} & \multicolumn{2}{|c|}{ Orgasmo } & \multicolumn{2}{|c|}{$\begin{array}{c}\text { Satis- } \\
\text { facción }\end{array}$} & \multicolumn{2}{|c|}{ Dolor } & \multicolumn{2}{|l|}{ Total } \\
\hline Edad & $\bar{x}$ & DS & $\bar{x}$ & DS & $\bar{x}$ & DS & $\bar{x}$ & DS & $\bar{x}$ & DS & $\bar{x}$ & DS & & \\
\hline$\leq 19$ años & 3.9 & \pm 1.2 & 4.4 & \pm 1.1 & 4.8 & \pm 1.2 & 4.7 & \pm 1.0 & 4.4 & \pm 1.4 & 5.0 & \pm 1.0 & 27.25 & \pm 5.52 \\
\hline 20 a 39 & 3.4 & \pm 1.2 & 4.0 & \pm 1.2 & 4.5 & \pm 1.1 & 4.4 & \pm 1.1 & 4.6 & \pm 1.3 & 4.7 & \pm 1.4 & 25.62 & \pm 5.59 \\
\hline 40 a 64 & 3.3 & \pm 1.1 & 3.8 & \pm 1.2 & 4.4 & \pm 1.1 & 4.2 & \pm 1.1 & 4.3 & \pm 1.3 & 4.3 & \pm 1.4 & 24.21 & \pm 5.60 \\
\hline$\geq 65$ años* & 3.6 & & 3.0 & & 3.0 & & 3.6 & & 4.0 & & 2.8 & & 20.00 & \\
\hline \multicolumn{15}{|l|}{ Estado civil } \\
\hline Soltera & 3.5 & \pm 1.1 & 4.0 & \pm 1.1 & 4.5 & \pm 1.1 & 4.4 & \pm 1.1 & 4.7 & \pm 1.1 & 4.8 & \pm 1.3 & 26.10 & \pm 5.14 \\
\hline Casada & 3.4 & \pm 1.2 & 4.0 & \pm 1.3 & 4.4 & \pm 1.2 & 4.2 & \pm 1.2 & 4.3 & \pm 1.3 & 4.3 & \pm 1.4 & 24.51 & \pm 6.21 \\
\hline Viuda & 3.5 & \pm 1.3 & 4.0 & \pm 1.2 & 4.6 & \pm 1.1 & 4.3 & \pm 1.2 & 4.4 & \pm 1.4 & 4.7 & \pm 1.4 & 25.52 & \pm 5.60 \\
\hline Divorciada & 3.0 & \pm 1.4 & 3.8 & \pm 1.3 & 4.7 & \pm 1.1 & 4.2 & \pm 0.9 & 4.4 & \pm 1.4 & 4.4 & \pm 0.9 & 24.44 & \pm 5.24 \\
\hline \multicolumn{15}{|l|}{ Religión } \\
\hline Católica & 3.5 & \pm 1.2 & 4.0 & \pm 1.2 & 4.5 & \pm 1.1 & 4.3 & \pm 1.1 & 4.5 & \pm 1.3 & 4.6 & \pm 1.4 & 25.34 & \pm 5.76 \\
\hline Evangélica & 3.4 & \pm 1.2 & 4.4 & \pm 0.9 & 5.1 & \pm 0.7 & 4.8 & \pm 1.2 & 4.4 & \pm 1.2 & 4.0 & \pm 1.4 & 26.08 & \pm 4.10 \\
\hline $\begin{array}{l}\text { Testigo de } \\
\text { Jehová }\end{array}$ & 2.0 & \pm 0.3 & 2.5 & 0.2 & 3.8 & \pm 0.5 & 2.9 & \pm 1.4 & 3.2 & \pm 2.4 & 4.8 & \pm 0.0 & 19.23 & \pm 3.80 \\
\hline Ninguna & 3.6 & \pm 1.1 & 4.3 & \pm 1.3 & 4.6 & \pm 1.3 & 4.4 & \pm 0.9 & 4.7 & \pm 1.0 & 4.9 & \pm 1.1 & 26.55 & \pm 4.34 \\
\hline Otras & 2.4 & \pm 1.7 & 2.6 & \pm 1.3 & 4.3 & \pm 1.5 & 3.7 & \pm 1.3 & 3.5 & \pm 1.5 & 4.8 & \pm 1.0 & 21.23 & \pm 6.06 \\
\hline
\end{tabular}




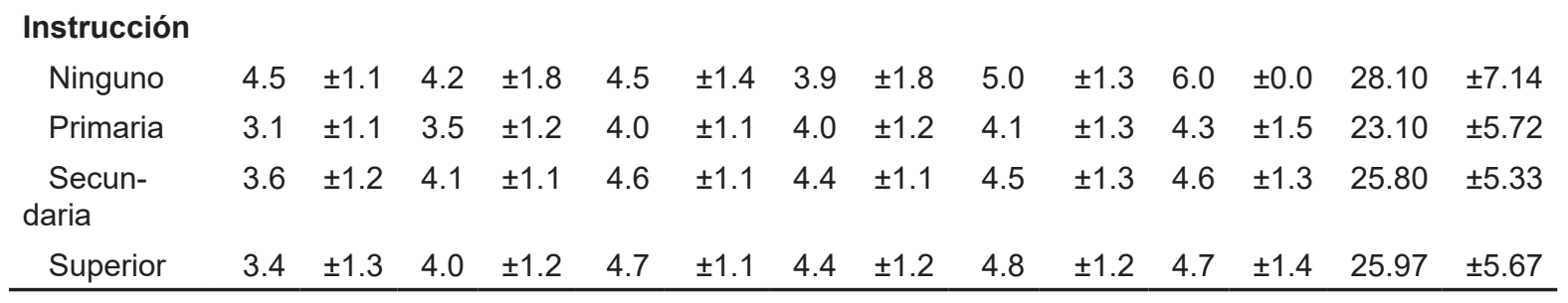

*En la investigación sólo participó una paciente con edad mayor a 65 años

Según características sociodemográficas se puede observar en la tabla 3 que los puntajes más bajos en relación a la media del IFSF sugerentes a disfunción sexual femenina, fueron más relevantes en dominios del deseo, todos los grupos de las variables sociodemográficas, observándose una tendencia inferior en los rangos de edad de 20 a 39 años (media 3.4, DS \pm 1.2 ), así como las mujeres divorciadas (media 3.0, DS \pm 1.4 ), religión Testigo de Jehová (media 2.0, DS \pm 0.3 ) e instrucción primaria (media 3.1, DS \pm 1.1 ).

Las pacientes que refirieron ser testigos de Jehová repitieron la tendencia de alteración en el IFSF en los dominios de: excitación, orgasmo y satisfacción con medias iguales o inferiores a 3.2; de la misma forma personas que indicaron ser de otras religiones mostraron cifras similares (Tabla $\mathrm{N}^{\circ} 3$ ).

Respecto a la puntuación final de la escala de IFSF, se consideró que los grupos que presentaron puntuación menor sugerente con disfunciones sexuales fueron: los grupos de edades entre 40 a 64 años (media 24.21, DS \pm 5.6 ), las personas divorciadas (media 24.44, DS \pm 5.6 ), las mujeres cuya religión era Testigo de Jehová (media 19.23, DS \pm 3.80$)$ y aquellas con un nivel de instrucción primaria (media 23.10, DS \pm 5.72 ) (Tabla $N^{\circ} 3$ ).

\section{Tabla $\mathrm{N}^{\circ} 4$}

Prevalencia de Disfunción Sexual Femenina en 253 pacientes atendidas en la comunidad de Sayausí, año 2019

\begin{tabular}{lllc}
\hline & $\mathbf{n}$ & $\%$ & IC 95\% \\
\hline Sí & 147 & 58.1 & \\
No & 106 & 41.9 & $51.82-64.38$ \\
\hline
\end{tabular}

En la Tabla $\mathrm{N}^{\circ} 4$ se puede observar que la prevalencia de disfunción sexual femenina en la población de estudio fue de $58.1 \%$ con un intervalo de confianza de $51.82 \%$ al $64.38 \%$.

\section{Tabla $N^{\circ} 5$}

Prevalencia de disfunción sexual en los dominios: deseo, excitación, lubricación, orgasmo, satisfacción, dolor en 253 pacientes atendidas en la comunidad de Sayausí, año 2019

\begin{tabular}{lrr}
\hline & n & \multicolumn{1}{c}{$\%$} \\
\hline Dominio deseo & & \\
$\quad$ Función sexual alterada & 177 & 70 \\
$\quad$ Función sexual normal & 76 & 30 \\
Dominio excitación & & \\
$\quad$ Función sexual alterada & 106 & 41.9 \\
$\quad$ Función sexual normal & 147 & 58.1 \\
Dominio lubricación & & \\
$\quad$ Función sexual alterada & 70 & 27.7 \\
$\quad$ Función sexual normal & 183 & 72.3 \\
Dominio orgasmo & & \\
$\quad$ Función sexual alterada & 94 & 37.2 \\
$\quad$ Función sexual normal & 159 & 62.8 \\
Dominio satisfacción & & \\
$\quad$ Función sexual alterada & 73 & 28.9 \\
$\quad$ Función sexual normal & 180 & 71.1 \\
Dominio dolor & & \\
$\quad$ Función sexual alterada & 79 & 31.2 \\
$\quad$ Función sexual normal & 174 & 68.8 \\
\hline
\end{tabular}

El $70 \%$ de mujeres encuestadas presentaron alteración de la función sexual en el dominio del deseo, el $41.9 \%$ en el de excitación y el $37.2 \%$ en el orgasmo. En menor frecuencia se alteraron los dominios de dolor, satisfacción y lubricación con el $31.2 \%, 28.9 \%$ y $27.7 \%$ respectivamente (Tabla $\mathrm{N}^{\circ} 5$ ). 


\section{DISCUSIÓN}

La sexualidad es sin duda uno de los pilares fundamentales en el bienestar de la mujer y su calidad de vida, no obstante, a pesar de ello no es usual que una mujer consulte por manifestaciones, cambios y/o alteraciones afines a su sexualidad, sobre todo por las barreras culturales, mitos, temores, creencias religiosas, así como su bajo nivel de educación; además, de la falta de formación y capacitación de los profesionales de salud para detectar y abordar dichos trastornos, entre otros factores que contribuyen a que el tema de disfunciones sexuales sea un tema silencioso ${ }^{21}$.

En la investigación participaron 253 mujeres, en su mayoría con rangos de edad entre 20 a 39 años, instrucción secundaria, de nivel socioeconómico medio y bajo, residentes en áreas urbanas, de estado civil solteras y religión católica. El presente estudio reveló como resultado una prevalencia de $58.1 \%$, sobre todo en mujeres con rangos de edad de 20 a 39 años, divorciadas, religión Testigo de Jehová e instrucción primaria, quienes pertenecen a esta religión, además presentan afecciones en dominios como: excitación, orgasmo y satisfacción.

Estas cifras son similares a las conclusiones de autores como: Shifren et $\mathrm{al}^{22}$, Choi y cols $^{23}$ y Bustos y Pérez ${ }^{24}$, quienes encontraron una prevalencia de disfunción sexual femenina de $43.1 \%$ y $46.1 \%$ y $41.6 \%$ respectivamente, al igual que Espitia ${ }^{1}$ con un $46.96 \%$. Prevalencias superiores las reportaron Carranza y Casillas $^{25}$, quienes concluyeron una frecuencia del $62.1 \%$ de DSF en premenopáusicas y $62.5 \%$ en posmenopáusicas, al igual que $\mathrm{Ma}$ y $\mathrm{cols}^{26}$, con un $72.7 \%$ y Singh y cols ${ }^{27}$ en un $73.2 \%$. Otros autores tales como Zhang y cols ${ }^{20}$, obtuvieron resultados inferiores con un $29.7 \%$ y Mostafa y cols ${ }^{28}$ en un $28 \%$.

De acuerdo a las formas clínicas de disfunción sexual o dominios, en nuestro estudio el $70 \%$ de mujeres encuestadas presentaron alteración en el deseo sexual, al igual que nuestro estudio Shifren et $\mathrm{a}^{22}$, encontraron que el dominio que se alteró con mayor frecuencia fue el deseo sexual con $28.6 \%$. Zhang y cols ${ }^{20}$ con $21.6 \%$ (IC del 99\% = 20.9-22.2). Ma y cols ${ }^{26}$ evidenciaron que trastorno de deseo, se presentó en un $37.6 \%$, de igual manera Wolpe y cols ${ }^{29}$ concluyeron que dicha alteración osciló entre $11 \%$ y el $75 \%$. Mientras Espitia ${ }^{1}$, indica que en su estudio se obtuvo un $75 \%$.
En relación a los otros dominios correspondientes a la disfunción sexual, en el presente estudio, la presencia de alteración en la excitación sexual se encontró en el $41.9 \%$, seguida de la alteración del orgasmo con el $37.2 \%$, en menor frecuencia se alteraron los dominios de dolor, satisfacción y lubricación con el $31.2 \%, 28.9 \%$ y $27.7 \%$ respectivamente. Los resultados que encontramos en la literatura médica son muy variados, es así que Shifren et $\mathrm{al}^{22}$, encontró que el $21.5 \%$ (IC del $99 \%=20.8-22.2)$ de pacientes tuvieron trastorno de excitación, el 18.9\% (IC del 99\% = 18.3-19.6) de la lubricación, $27.9 \%(99 \% \mathrm{Cl}=27.2-28.7)$ del orgasmo y $14.1 \%(99 \% \mathrm{Cl}=13.6-14.7)$ dolor sexual. Hayes y cols ${ }^{30}$ mostraron un $64 \%$ de mujeres con alteración en la excitación, dificultad en el orgasmo en el $35 \%$ y dolor sexual en el $26 \%$. Singh y cols ${ }^{27}$ evidenciaron que el $96.6 \%$ presentaban alteraciones en la lubricación, 91.3\% excitación; $86.6 \%$ en el trastorno del orgasmo, $77.2 \%$ en deseo y dolor sexual con $64.4 \%$. Halle y cols $^{31}$ demostraron en su estudio que un total de $171(42 \%)$ de 405 estudiantes sexualmente activos de la Universidad de Buea, África, presentaron: problemas de dolor sexual $46.9 \%$, orgasmo $42 \%$, deseo $29.1 \%$ y excitación $21.2 \%{ }^{31}$. Koops y cols ${ }^{32}$, indicaron que en su estudio predominó la categoría del dolor sexual, con una frecuencia entre el 3\% al $95.5 \%$. Ma y cols ${ }^{26}$, concluyeron que $36.8 \%$ tuvo trastorno del orgasmo, $30.6 \%$ dolor sexual, $25.4 \%$ lubricación y de $23.6 \%$ en la excitación. Mostafa y cols ${ }^{28}$, encontró un $69.3 \%$ presentó dolor sexual, seguido de $53.3 \%$ el trastorno de lubricación y $52 \%$ de excitación. Wolpe y cols ${ }^{29}$, observaron que la excitación se alteró entre $8 \%$ y el $68.2 \%$, alteración en dificultad para llegar al orgasmo de $18 \%$ y $55.4 \%$ y dispareunia de $1.2 \%$ a $56.1 \%$. Bustos y Pérez ${ }^{24}$, analizaron dentro de sus categorías que el porcentaje fue de $63.3 \%$ en el trastorno del orgasmo, $37 \%$ del deseo, $12.1 \%$ de excitación, $13.4 \%$ de problemas de satisfacción sexual, $11.4 \%$ de dispareunia y $9.5 \%$ de lubricación.

\section{CONCLUSIÓN}

La prevalencia de disfunción sexual femenina en las pacientes que acuden al Centro de Salud Sayausí, fue muy alta (58.1\%), de las pacientes que presentaron disfunción sexual, las características más frecuentes fueron el grupo de edad entre 20 a 39 años, estado civil divorciadas e instrucción primaria. 
De acuerdo a las diferentes formas clínicas de la disfunción sexual femenina, el deseo fue en $70 \%$, seguido de la excitación con un $41.9 \%$, el orgasmo fue de $37.2 \%$. En menor frecuencia se alteraron los dominios de dolor con un $32.2 \%$, satisfacción con un $28.9 \%$ y finalmente la lubricación con el $27.7 \%$.

\section{RECOMENDACIONES}

Es necesario que el personal sanitario y las autoridades del Centro de Salud Sayausí, sea capacitado y sensibilizado, no solamente en temas sobre disfunción sexual, sino en todo lo referente al campo de la sexualidad, pues resulta trascendental poner en evidencia la importancia de la temática planteada por las repercusiones en la calidad de vida de las pacientes. Además, es importante la planificación e implementación de estrategias de prevención y/o intervención de disfunciones sexuales, enfocadas en impulsar programas que fomenten una educación e información eficaz y efectiva, que oriente a una participación activa de la pareja y de la sociedad en general.

\section{ASPECTOS BIOETICOS}

El estudio fue aprobado por el Comité Institucional de Bioética en Investigaciones en seres humanos de la Universidad Católica de Cuenca, carrera de Medicina.

\section{INFORMACIÓN DE LOS AUTORES}

- Pinos Vélez Erika Aracely. Médica. Libre ejercicio. Cuenca-Azuay-Ecuador.

e-mail: eapv0612@hotmail.com

ORCID: https://orcid.org/0000-0002-0932-9991

- Picón Rodríguez Jennifer Pamela. Médica. Libre ejercicio. Cuenca-Azuay-Ecuador.

e-mail: jennifer2436@hotmail.com

ORCID: https://orcid.org/0000-0003-4264-6208

- Tapia Cárdenas Jeanneth Patricia. Médica. Especialista en Obstetricia y Ginecología. Magíster en Investigación de la Salud. Universidad Católica de Cuenca. Unidad Académica de Salud y Bienestar. Cuenca-Azuay-Ecuador.

e-mail: janethtapiac@hotmail.com

ORCID: https://orcid.org/0000-0003-3203-8225

\section{CONTRIBUCIÓN DE LOS AUTORES}

Todas las autoras contribuyeron de manera similar en la elaboración del presente artículo.

\section{CONFLICTO DE INTERESES}

Se declara no existencia de conflicto de intereses.

\section{FUENTES DE FINANCIAMIENTO}

Autofinanciado.

\section{BIBLIOGRAFÍA}

1. Espitia F. Evaluación de la prevalencia de disfunción sexual en mujeres médicos, del Eje Cafetero colombiano, en etapa de climaterio. Archmed. 2017;17(1):70-7. Disponible en: http://revistasum.umanizales. edu.co/ojs/index.php/archivosmedicina/ article/view/1897

2. Zuluaga J, Cruz J, Meneses M. Medicalización, promoción de la enfermedad y disfunción sexual femenina. RevCS. 2018;41-66. doi: 10.18046/recs.i24.2308

3. Chou D, Cottler S, Khosla R, Reed GM, Say L. Sexual health in the International Classification of Diseases: implications for measurement and beyond. Reproductive Health Matters. 2016;23(46):185-92.doi: 10.1016/j.rhm.2015.11.008

4. Guachamin M, Jacome I. Análisis de la disfunción sexual en las mujeres de 15 a 42 años asociado al uso del implante subdérmico en el Servicio de Obstetricia, en el Centro de Salud Sangolquí [Internet]. Universidad Central del Ecuador; 2017. Disponible en: http://www.dspace.uce.edu. ec/handle/25000/11828

5. Moyano N, Sierra J. Descifrando las disfunciones sexuales femeninas en el DSM5.2016;24(2):11.Disponible en: https://dialnet. unirioja.es/servlet/articulo?codigo $=5556511$

6. Murillo FH, Salonginos OD. Vademecum Sexual: Farmacos y Disfuncion Sexual Actualizacion. 2017;3(4):33.Disponible en: https://psicosomaticaypsiquiatria.com/ wp-content/uploads/2017/06/psicosom_ psiquiatr_201711_27-59.pdf

7. López S, Faro C, Lopetegui L, Pujol-Ribera E, Monteagudo M, Cobo J. et al. Impacto del abuso 
sexual durante la infancia-adolescencia en las relaciones sexuales y afectivas de mujeres adultas. Gaceta Sanitaria. 2016;31(3):210-9. doi: 10.1016/j.gaceta.2016.05.010

8. Figueroa L, Duarte G, Sánchez MB, Ruyman P. Abordaje de la sexualidad en atención primaria: ¿qué valorar? Revista Ene. 2015;9(2):2-9.doi: 10.4321/S1988-348X2015000200006

9. Organization WH. Measuring sexual health: conceptual and practical considerations and related indicators. 2010;(1):15-25.Disponible en: http://apps.who.int/iris/bitstream/ handle/10665/70434/who_rhr_10.12_eng. pdf;jsessionid=91AAA9A7Ë9E-516A13ACF52D897E8DE12?sequence $=1$

10. Farmer M, Yoon H, Goldstein I. Future Targets for Female Sexual Dysfunction. The Journal of Sexual Medicine. 2016;13(8):1147-65.doi: 10.1016/j.jsxm.2016.05.016

11. Kingsberg SA, Woodard T. Female Sexual Dysfunction: Focus on Low Desire. Obstetrics \& Gynecology. 2015;125(2):477-86.doi: 10.1097/AOG.0000000000000620

12. Basson R. Human sex-response cycles. J Sex Marital Ther. 2012;27(1):33-43.doi: 10.1080/00926230152035831

13. Masters $M$, Johnson V. Human Sexual Response. Health. 2017;6(10):33. doi: 10.4236/health.2014.610118

14. Tsui $\mathrm{KH}$, Wang $\mathrm{PH}$, Chen CK, Chen $\mathrm{YJ}$, Chiou SH, Sung YJ, et al. Non-classical estrogen receptors action on human dermal fibroblasts. Taiwanese Journal of Obstetrics and Gynecology. 2017;50(4):4748.Disponible en: https://linkinghub.elsevier. com/retrieve/pii/S1028455911001720

15. WangPH, ChaoHT, ChaoKC. Chemotherapyinduced Gonadotoxicity. Taiwanese Journal of Obstetrics and Gynecology. 2016;49(1):12.doi: $10.1016 / S 1028-4559(10) 60001-0$

16. Chen $\mathrm{C}-\mathrm{H}$, Lin $\mathrm{Y}-\mathrm{C}$, Chiu L-H, Chu Y-H, Ruan F-F, Liu W-M, et al. Female sexual dysfunction: Definition, classification, and debates. Taiwanese Journal of Obstetrics and Gynecology. 2016;52(1):3-7.doi: 10.1016/j. tjog.2013.01.002
17. Alcántara Montero A, Sánchez Carnerero $\mathrm{Cl}$. Disfunción sexualfemenina:opciones detratamiento farmacológico. Semergen. 2016;42(5):33-7.doi: 10.1016/j.semerg.2016.02.008

18. American Psychiatric Association, editor. Guía de consulta de los criterios diagnósticos del DSM-5. Arlington, VA: American Psychiatric Publishing; 2014. 438 p.Disponible: https://www.psychiatry.org/ psychiatrists/practice/dsm

19. Rodriguez A. Metodo de Estratificacion Social Graffar Mendez. 2016;3(4):23.doi: 10.13140/2.1.4633.2964

20. Zhang C, Tong J, Zhu L, Zhang L, Xu T, Lang $J$, et al. A Population-Based Epidemiologic Study of Female Sexual Dysfunction Risk in Mainland China: Prevalence and Predictors. The Journal of Sexual Medicine. 2017;14(11):1348-56.doi: jsxm.2017.08.012

21. Monterrosa-Castro Á, Márquez-Vega J, Arteta-Acosta C. Disfunción sexual en mujeres climatéricas afrodescendientes del Caribe Colombiano. 2014;27(2):11. Disponible en: http://www.scielo. org.co/scielo.php? pid=S0121$07932014000100004 \&$ script $=s c i$ abstract\&tlng=es

22. Shifren JL, Monz B, Russo P, Segreti A, Johannes C. Sexual problems and distress in United States women: prevalence and correlates. Obstet Gynecol. 2013;112(2):8908. doi: 10.1097/AOG.0b013e3181898cdb

23. Choi H, Kim J-H, Park J-Y, Shim J-S, Lee $\mathrm{J}-\mathrm{G}$, Yoon $\mathrm{H}-\mathrm{Y}$, et al. Assessment of sexual dysfunction and determination of its risk factors in the Republic of Korea. International Journal of Gynecology \& Obstetrics. 2015;125(1):604.doi: 10.1016/j.ijgo.2013.10.006

24. BustosB.G,PérezVR. Relaciónentrefactores psicosociales y el Índice de Función Sexual Femenina en población rural de la comuna de Teno, Región del Maule. Perinatología y Reproducción Humana. 2018;32(1):9-18.doi: 10.1016/j.rprh.2018.03.014

25. Carranza-Lira S, Núńez F de DC. Sexual dysfunction prevalence in a group of preand postmenopausal Mexican women. pm. 2018;17(1):39-42.doi: 10.5114/ pm.2018.74901 
26. Ma J, Pan L, Lei Y, Zhang A, Kan Y. Prevalence of Female Sexual Dysfunction in Urban Chinese Women Based on Cutoff Scores of the Chinese Version of the Female Sexual Function Index: A Preliminary Study. The Journal of Sexual Medicine. 2015;11(4):90919.doi: $10.1111 / \mathrm{jsm} .12451$

27. Singh J, Tharyan P, Kekre N, Singh G, Gopalakrishnan G. Prevalence and risk factors for female sexual dysfunction in women attending a medical clinic in south India. - J Postgrad Med. 2014;55(2):113-20.

28. Mostafa AM, Khamis Y, Helmy HK, Arafa $\mathrm{AE}$, Abbas AM. Prevalence and patterns of female sexual dysfunction among overweight and obese premenopausal women in Upper Egypt; a cross sectional study. Middle East Fertility Society Journal. 2017;50(5):100-25. doi: 10.1016/j.mefs.2017.08.006

29. Wolpe RE, Zomkowski K, Silva FP, Queiroz APA, Sperandio FF. Prevalence of female sexual dysfunction in Brazil: A systematic review. European Journal of Obstetrics \& Gynecology and Reproductive Biology. 2017;211(3):26-32.doi: 10.1016/j. ejogrb.2017.01.018

30. Hayes RD, Bennett CM, Fairley CK, Dennerstein L. What can Prevalence Studies Tell Us about Female Sexual Difficulty and Dysfunction? The Journal of Sexual Medicine. 2013;3(4):589-95.Disponible: https://linkinghub.elsevier.com/retrieve/pii/ S1743609515313564

31. Halle Ekane GE, Timti LF, Tanue EA, Ekukole CM, Yenshu EV. Prevalence and Associated Factors of Female Sexual Dysfunction Among Sexually Active Students of the University of Buea. Sexual Medicine. 2019;9(5):10.doi: 10.1016/j.esxm.2021.100402

32. Koops TU, Briken P. Prevalence of Female Sexual Function Difficulties and Sexual Pain Assessed by the Female Sexual Function Index: A Systematic Review. The Journal of Sexual Medicine.2018;15(11):1591-9.doi: 10.1016/j.jsxm.2018.09.005 